\title{
Accumulation of Fetal Fibronectin mRNAs during the Development of Rat Cardiac Hypertrophy Induced by Pressure Overload
}

\author{
Jane Lyse Samuel, Alice Barrieux, Sylvie Dufour, * Isabelle Dubus, Francis Contard, Victor Koteliansky, * \\ Farahnaz Farhadian, Françoise Marotte, Jean-Paul Thiéry, and Lydie Rappaport \\ U127 INSERM, Hopital Lariboisière, 75010 Paris; and *URA 230, Ecole Normale Supérieure 75230 Paris Cedex 05, France
}

\begin{abstract}
Cardiac pressure overload induces a shift towards the fetal form of major proteins expressed by the myocytes, and an accumulation of extracellular matrix proteins. One of them, fibronectin (FN), accumulates soon after the imposition of pressure overload. Because FN exists both as cellular FN (c-FN) locally synthesized by nonmuscle cells and as "plasma-FN" (p-FN) synthesized by the hepatocytes, the first issue of this study was to determine whether FN accumulation within the myocardium in response to pressure overload is paralleled by a local increase in mRNA. The expression of c-FN isoforms being developmentally regulated in a tissue-specific manner, the types of FN exons expressed by cardiac cells were analyzed. Pressure overload was induced in 25-d-old rats by stenosis of the thoracic aorta. Using in situ hybridization, we show that the mRNAs encoding the fetal forms of $\mathrm{c}-\mathrm{FN}$ are accumulated in the interstitial tissue of fetal rat hearts but are absent in adult. 1-3 d after aortic stenosis, the fetal forms of c-FN mRNAs were found in the wall of coronary arteries and in focal areas of the myocardium. Thus nonmuscle cells and smooth muscle cells, like myocytes, do respond to pressure overload by reexpressing fetal gene transcripts. (J. Clin. Invest. 1991. 88:17371746.) Key words: aortic stenosis • myocardium • in situ hybridization • EIIIA-fibronectin • EIIIB-fibronectin
\end{abstract}

\section{Introduction}

Fibronectin (FN) ${ }^{1}$ is one of the major components of the extracellular matrix in the heart (1). It is deposited as an insoluble complex of either a cellular form (c-FN) which is synthesized and secreted locally by the nonmuscle cells or a circulating form (plasma-FN, p-FN) synthesized and secreted by hepatocytes (2-5). The polypeptide is composed of three kinds of repeats: types I, II, and III $(6,7)$. The domains homologous to types I and II are common to c-FN and p-FN. Multiple forms of FN arise by the alternative splicing of a primary transcript originating from a single gene (reviews in 6,7 ). Sequence variations may occur at three points of the FN subunit: extra domains $A$ and $B$ (EDA and EDB, respectively) and IIICS in human fibronectin equivalent to EIIIA, EIIIB, and V in the rat

Received for publication 25 February 1991 and in revised form 11 June 1991.

1. Abbreviations used in this paper: c-FN, cellular form fibronectin; EDA, extra domain A; EDB, extra domain B; $p-F N$, plasma fibronectin.

J. Clin. Invest.

(c) The American Society for Clinical Investigation, Inc

$0021-9738 / 91 / 11 / 1737 / 10 \$ 2.00$

Volume 88, November 1991, 1737-1746 proteins $(6,8,9)$. The c-FN forms may include the A and $\mathrm{B}$ domains which are always absent from the p-FN (6). The A and $B$ domains are present in c-FN synthesized during embryonic development $(10,11)$. Their expression has been shown to be regulated during fetal and postnatal development of the human smooth muscle cells and is organ-specific (12).

Using immunohistochemistry, we have demonstrated that the accumulation of fibronectin is a very sensitive marker of cardiac ischemic lesions $(13,14)$. In addition, c-FN deposits have recently been shown to occur within $3 \mathrm{~d}$ after coronary occlusion in the zone of rat myocardial infarction, the accumulation of p-FN preceeding that of c-FN (13).

The development of cardiac hypertrophy, that characterizes the adaptation of myocardium to pressure overload, is associated with changes in genomic expression of the myocytes that consist mainly in a shift towards the fetal forms of a series of multigenic families (reviewed in 15, 16). Among the early responses of the myocardium to overload is a prompt rise in the accumulation of the main constituents of the extracellular matrix, fibronectin and collagens I-III and IV $(14,17)$. It is thought that these changes in the extracellular matrix depend mostly on the nonstriated cardiac cells (endothelial cells, fibroblasts, and smooth muscle cells) which are known to synthesize the majority of the extracellular matrix proteins in the heart $(18,19,20)$. This leads to the following questions: $(a)$ Does the accumulation of fibronectin within the myocardium in response to pressure overload correlate with an accumulation of mRNA? (b) Are the embryonic FN mRNA isoforms reexpressed in response to pressure overload?

To address these questions, we have used specific cRNA probes encoding the amino terminus of FN (FN-I) common to all fibronectins and to the EIIIA and EIIIB exons typical of c-FN for in situ hybridization of fetal and hypertrophied rat myocardium.

We show that (a) EIIIA- and EIIIB-containing mRNAs are expressed in the fetal heart but are absent in adult; $(b)$ there is an early accumulation of the fetal FN mRNAs in necrotic areas and in the wall of coronary arteries of adult hearts submitted to pressure overload. This is the first demonstration that nonmuscle cells and smooth muscle cells can reexpress fetal gene transcripts (FN-EIIIA ${ }^{+},-$EIIIB $^{+}$) during the process of cardiac hypertrophy induced by pressure overload.

\section{Methods}

Animals. All studies were performed on Wistar rats (Iffacredo, Lyon, France) which were kept under controlled housing conditions.

Female rats were sacrificed at $19 \mathrm{~d}$ postcoitum, and fetuses were removed and killed by decapitation.

Cardiac overload was produced in 25-d-old female rats (body wt, $60 \pm 5 \mathrm{~g}$ ) by coarctation of the ascending aorta with a partially occluded Weck hemoclip (21). The same operation was performed on shamoperated animals except that no clip was placed around the aorta. Five 
animals were killed 1 and $3 \mathrm{~d}$ after surgery (four operated and one sham-operated animals for each day). The hearts were dissected, trimmed free of large vessels, and weighed.

Preparation of probes for in situ hybridization. The vectors used in this study were either pSPT 18 or pSPT 19 from Boehringer-Mannheim GmbH, Mannheim, Germany. These two vectors differ only by the orientation of the multiple cloning site cassette. Subcloning and in vitro transcriptions are summarized in Fig. 1. The Sau III-Hpa II cDNA fragment encoding the ED1 segment of human FN (FN-EIIIA) was excised from the pFH111 clone (22), the $3^{\prime}$ ends were blunted and the fragment was inserted by blunt ligation in the filled Bam HI and Acc I sites of pSPT 19. The Hpa II-Hind III cDNA fragment encoding the ED 2 segment of human FN (FN-EIIIB) was excised from a cDNA clone kindly provided by Dr. L. Zardi. After fill-in, the fragment was inserted by blunt ligation in the blunted Acc I and Hind III sites of pSPT 18. The Sna BI-Sty I cDNA fragment encoding the signal peptide, the propeptide and the first type I homology segment of human FN (FN-I) was excised from a full-length $\mathrm{hFN}$ cDNA expression vector (23). The staggered $3^{\prime}$ end of the purified fragment was filled in and the fragment was inserted by blunt ligation in the filled Eco RI and blunt Sma I sites of pSPT 19.

Before synthesis of cRNA, FN-I was linearized with Bam HI, FNEIIIA with Eco RI and FN-EIIIB with Hind III. Control RNA was synthesized from FN-I linearized with Eco RI (Fig. 1). Single-strand cRNA or RNA probes were synthesized in the presence of ${ }^{35}$ S]UTP $\alpha S$ (NEN Research Products; Dupont, Wilmington, DE) and either T7 or SP6 RNA polymerases from a commercially available transcription kit (Boehringer Mannheim GmbH). The synthesized RNA probes were separated from the nonincorporated ribonucleosides by repeated ethanol precipitation and used without prior alkaline treatment. The probes were diluted to a final concentration of $\sim 60,000 \mathrm{cpm} / \mu \mathrm{l}$ in $50 \%$ formamide, $0.3 \mathrm{M} \mathrm{NaCl}, 20 \mathrm{mM}$ Tris- $\mathrm{HCl}$ (pH 8), $5 \mathrm{mM}$ EDTA, $10 \mathrm{mM}$ sodium phosphate $(\mathrm{pH} 8), 10 \%$ dextran sulfate, $1 \times$ Denhart's, $0.5 \mathrm{mg} / \mathrm{ml}$ yeast RNA, and $20 \mathrm{mM}$ dithiothreitol (DTT).

Preparation and prehybridization of tissue sections. Two protocols were used indifferently to fix and embed fetal and adult hearts. Briefly, hearts were fixed overnight in 4\% paraformaldehyde in PBS, dehydrated, and infiltrated with paraffin as previously described (24); alternatively they were fixed in $2 \%$ paraformaldehyde in PBS for $2 \mathrm{~h}$ at $4^{\circ} \mathrm{C}$ then washed in PBS containing 30\% sucrose for $4 \mathrm{~h}$ at $4^{\circ} \mathrm{C}$, embedded in OCT (RUA, Torcy, France), and frozen in isopentane precooled with liquid nitrogen.

Serial paraffin sections ( $5 \mu \mathrm{m}$ thick) were transferred to chrome alun gelatin-coated slides, and stored dry at $4^{\circ} \mathrm{C}$ until they were used for in situ hybridization. Serial cryosections ( $5 \mu \mathrm{m}$ thick) were dried a few minutes at room temperature, fixed in $4 \%$ paraformaldehyde for 5 min, washed in PBS $(2 \times 5 \mathrm{~min})$, then dehydrated in ethanol and stored at $-70^{\circ} \mathrm{C}$ with dessicant until used for in situ hybridization.
Procedures before hybridization were similar to those described by Wilkinson et al. (25). The paraffin sections were deparaffinized in xylene, afterwards both paraffin and cryosections were treated under identical conditions: i.e., rehydrated, fixed with $4 \%$ paraformaldehyde in PBS, digested with proteinase $K$, postfixed, treated with triethanolamine/acetic anhydride, washed, and dehydrated.

Hybridization and washing procedures. All probes were of similar specific activities and length $(237,213$, and 257 NT, respectively), and were used at the same concentrations in the hybridization mixture. Approximately $7 \mu \mathrm{l}$ of hybridization mixture were applied onto each section and the slides were incubated at $50^{\circ} \mathrm{C}$ overnight. After washing at $50^{\circ} \mathrm{C}$ in $5 \times \mathrm{SSC}$ and $10 \mathrm{mM}$ DTT, sections were subjected to stringent washing at $65^{\circ} \mathrm{C}$ in $50 \%$ formamide, $2 \times \mathrm{SSC}, 10 \mathrm{mM} \mathrm{DTT}$. They were then washed in Tris-EDTA buffer before treatment with $20 \mu \mathrm{g} / \mathrm{ml}$ RNAse A for $30 \mathrm{~min}$ at $37^{\circ} \mathrm{C}$. After washes in $2 \times \mathrm{SSC}$ and $0.1 \times \mathrm{SSC}$ at room temperature for $15 \mathrm{~min}$, the sections were dehydrated, dipped in Kodak NTB2 nuclear track emulsion (Eastman Kodak Co., Rochester, $\mathrm{NY}$ ), and exposed for $12 \mathrm{~d}$ in light-tight boxes with dessicant at $4^{\circ} \mathrm{C}$. Sections were developed in Kodak D19, mounted, and analyzed using both light- and dark-field optics of a Leitz Dialux microscope.

The hybridization conditions and the specificity of the RNA probes were tested on fetal and adult rat cardiac tissues. Labeled mRNA synthesized from the FN-I plasmid was used as a negative control probe.

\section{Results}

FN gene expression in fetal and adult rat heart. Fibronectin mRNAs present in cardiac tissue were localized by hybridization of the sections with ${ }^{35} \mathrm{~S}$-labeled RNA probes complementary to ED1, ED2, and type I sequences (FN-EIIIA, FN-EIIIB, and FN-I in Fig. 1). These sequences are highly conserved between human and rat $(7,26)$; therefore the human probes were used to detect rat mRNAs under stringent conditions.

When FN-I, -EIIIA, and -EIIIB probes were hybridized to serial sections of fetal rat heart, positive signals were obtained with all three probes (Fig. 2). At this low magnification, the inner part of both the left and right ventricles as well as the epicardium were strongly labeled and the distribution of FN-I and -EIIIA mRNAs were identical (Fig. 2, $a$ and $b$ ). The labeling pattern obtained for FN-EIIIB mRNAs differed slightly from those of the two other forms of mRNAs throughout the entire cardiac section: FN-EIIIB signal was less abundant than that of FN-I and -EIIIA in the left ventricle while all three were similar in the right ventricle. Fig. 2 also demonstrates the preferential accumulation of these mRNAs in the ventricular trabecu-

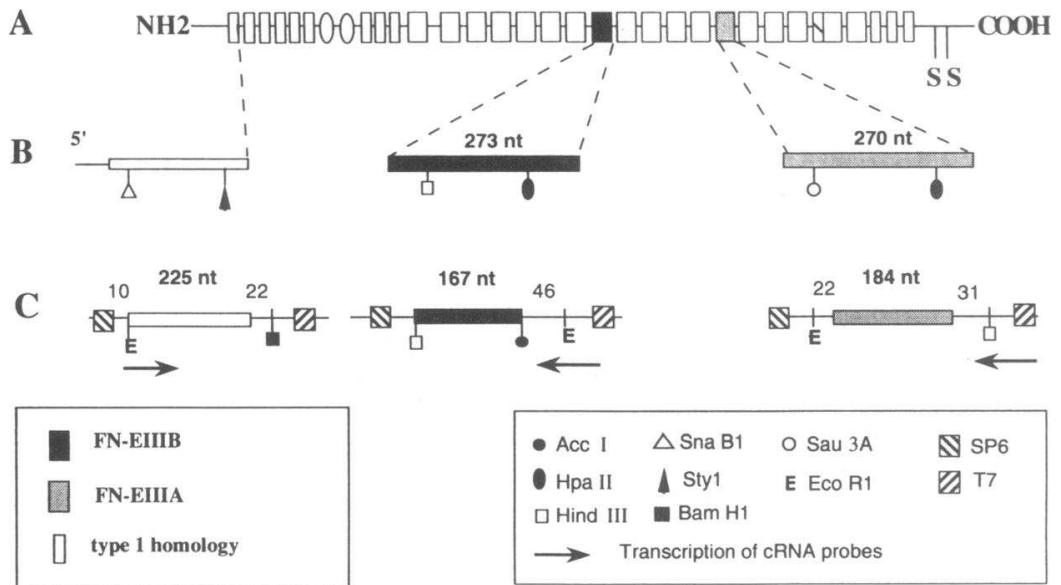

Figure 1. $(A)$ Diagram showing the structure of fibronectin and extradomains EIIIA and EIIIB subjected to differential splicing during FN RNA maturation. $(B)$ cDNA fragments encoding FN-I representing the signal peptide, the propeptide and the first type I homology domain, EIIIA representing extradomain I and EIIIB representing extradomain 2 of fibronectin (ED1 and ED2 in human nomenclature). (C) FN cDNA subclones used to synthesize the riboprobes; the size of FN and plasmid sequences included in the final transcripts, the promotor regions and the direction of cRNA transcription are indicated. 

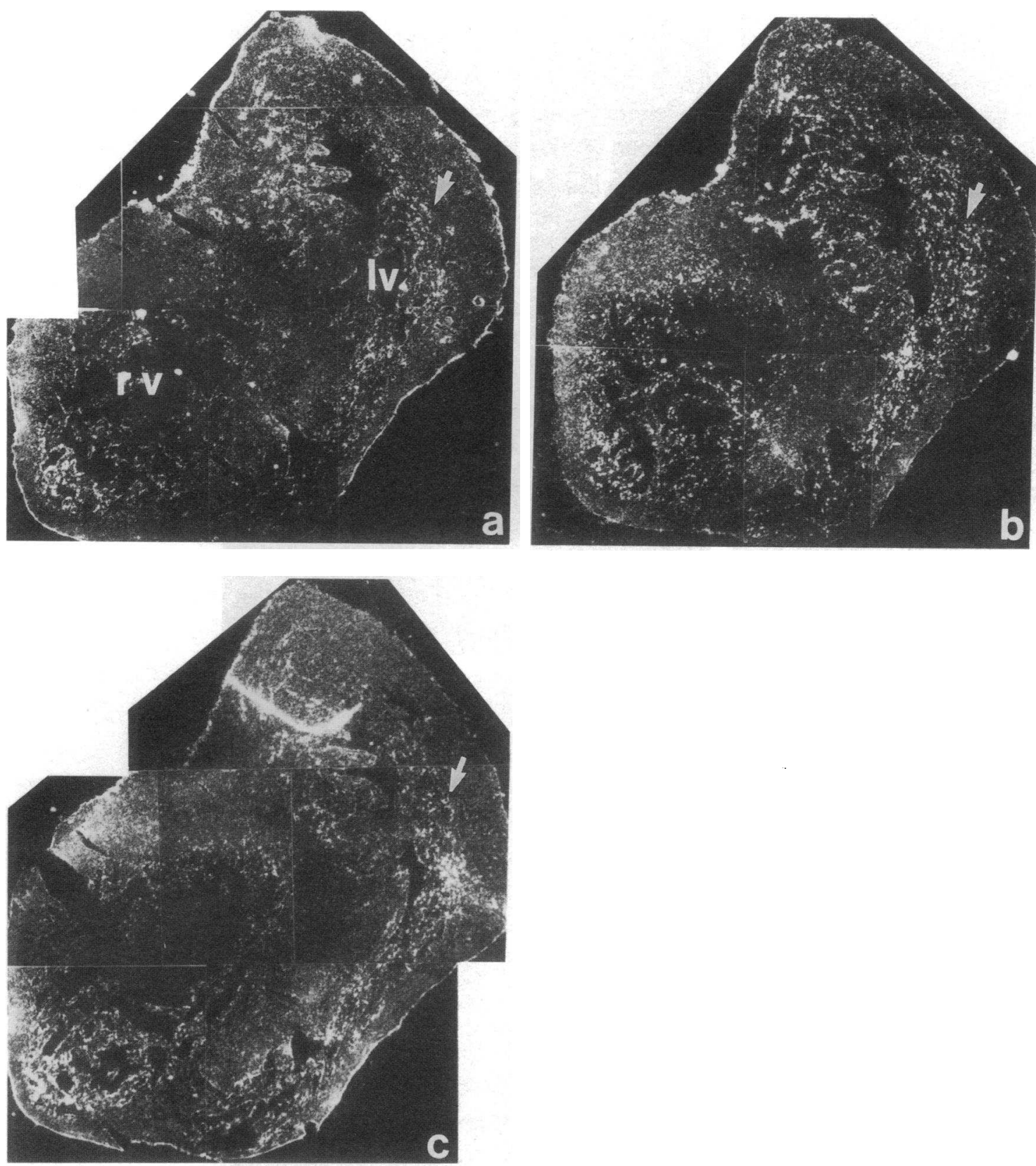

Figure 2. FN gene expression in the fetal rat heart. Montage of micrographs from whole ventricular sections hybridized with FN-I (a), EIIIA (b), and EIIIB probes $(c)$. Note that areas of the left myocardium express less FN-EIIIB mRNAs than FN-I and -EIIIA mRNAs (arrow).

lae of the fetal heart; only a small signal was detected in the midwall of both ventricles.

The examination of these sections at a higher magnification allowed us to define more precisely the localization of FN mRNAs within the fetal myocardium (Fig. 3). In the right ventricle, the positive signals obtained with each of the three probes appeared as scattered dots randomly distributed within the trabeculae and their respective patterns of distribution were very similar. When the distribution of FN-EIIIB ${ }^{+}$mRNAs obtained with dark-field optics was compared to the phase contrast image, it was evident that the silver grains were localized mainly over areas rich in nonmuscle cells such as endothelial cells and fibroblasts and virtually absent from the myocardial areas enriched in striated myocytes. Consequently FN-EIIIA ${ }^{+}$ and -EIIIB ${ }^{+}$mRNAs were abundantly expressed during late fetal development and restricted mostly to nonmyocytes. In the 26-d-old rat heart when the developmental growth of the heart is almost completely finished, neither FN-EIIIA nor FNEIIIB mRNAs were detected in the myocardium (Fig. 4, $d$ and $f$ ). Only a slight but significant signal was found in the media of coronary arteries with the FN-I probe (Fig. 4 b).

FN gene expression after thoracic aortic stenosis. Thoracic aortic stenosis provides a well-defined and reproducible experimental model of cardiac hypertrophy $(24,27) .1 \mathrm{~d}$ after surgery, the degree of hypertrophy is rather small (12\%). As shown in Figs. 4 and 5, at this stage the major changes detected in FN mRNA accumulation occurred in the blood vessels. Each probe generated a strong positive signal in the media of arteries 

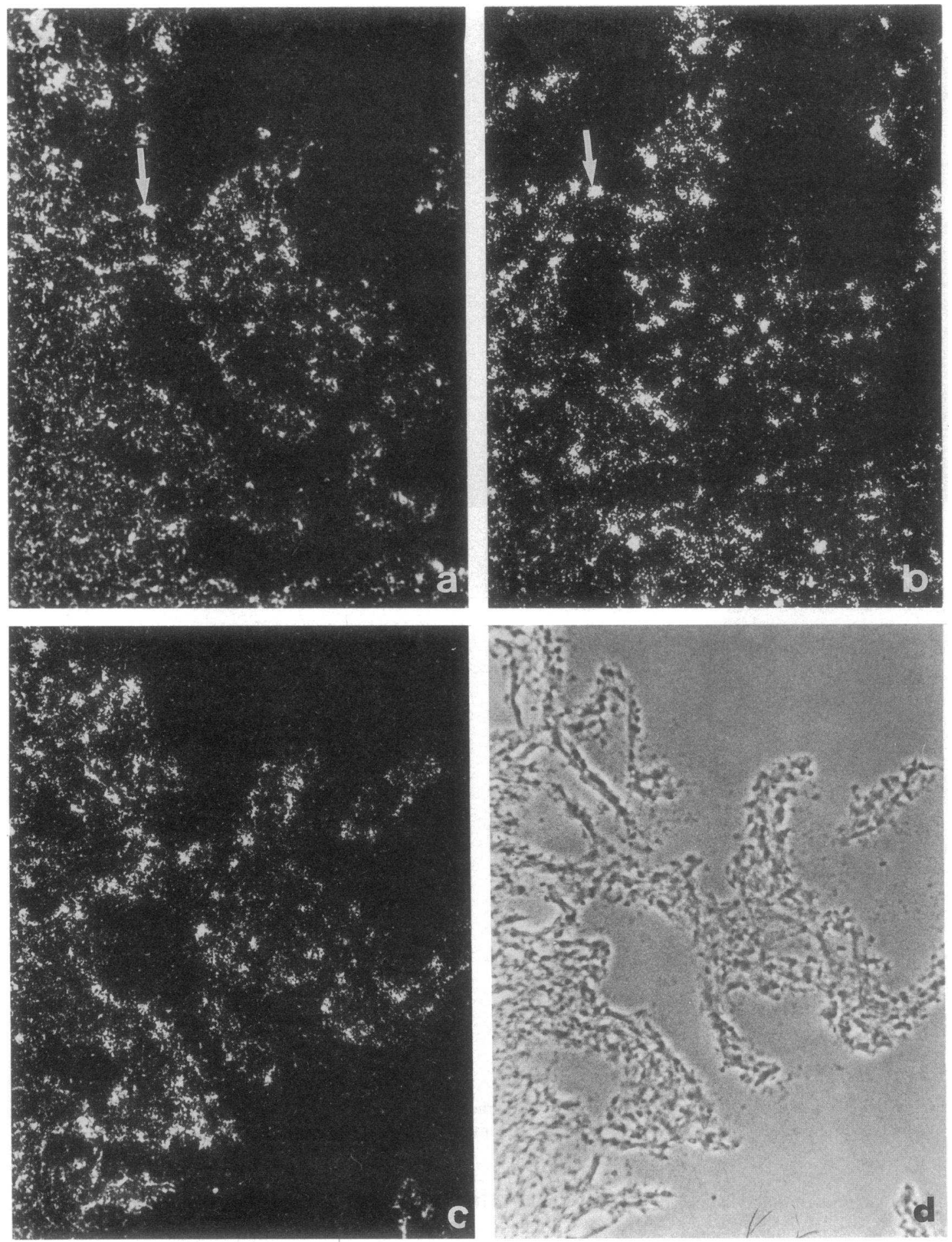

Figure 3. Colocalizations of FN-EIIIA and -EIIIB mRNAs within the trabeculae of the right ventricle seen at higher magnification. The sections were hybridized with FN-I $(a)$, -EIIIA $(b)$, and -EIIIB $(c)$ probes; phase contrast image $(d)$. High grain densities are seen in nonmuscle cells such as endothelial cells (arrows).

regardless of their size or their location in both the left and right ventricles, the accumulation of FN-EIIIB ${ }^{+}$mRNAs being particularly striking. No specific labeling was observed in either the adventitial tissue (Figs. 4 and 5) or the walls of veins (Fig. 5). The myocardium surrounding the vessels did not react with the FN-EIIIB probe (Figs. $4 e$ and $5 b$ ) but was reactive with FN-I and FN-EIIIA probes. The specific labeling appeared as dots scattered throughout the muscle layer; the hybridization signal, which was not localized over the myocytes, most probably corresponded to the interstitial tissue (Figs. 4 and 5). One of the four hearts studied was completely unreactive with the FN probes; the other three exhibited the characteristic pattern of FN mRNA accumulation described in Figs. 4 and 5. Cardiac sections from sham-operated animals did not hybridize to the 

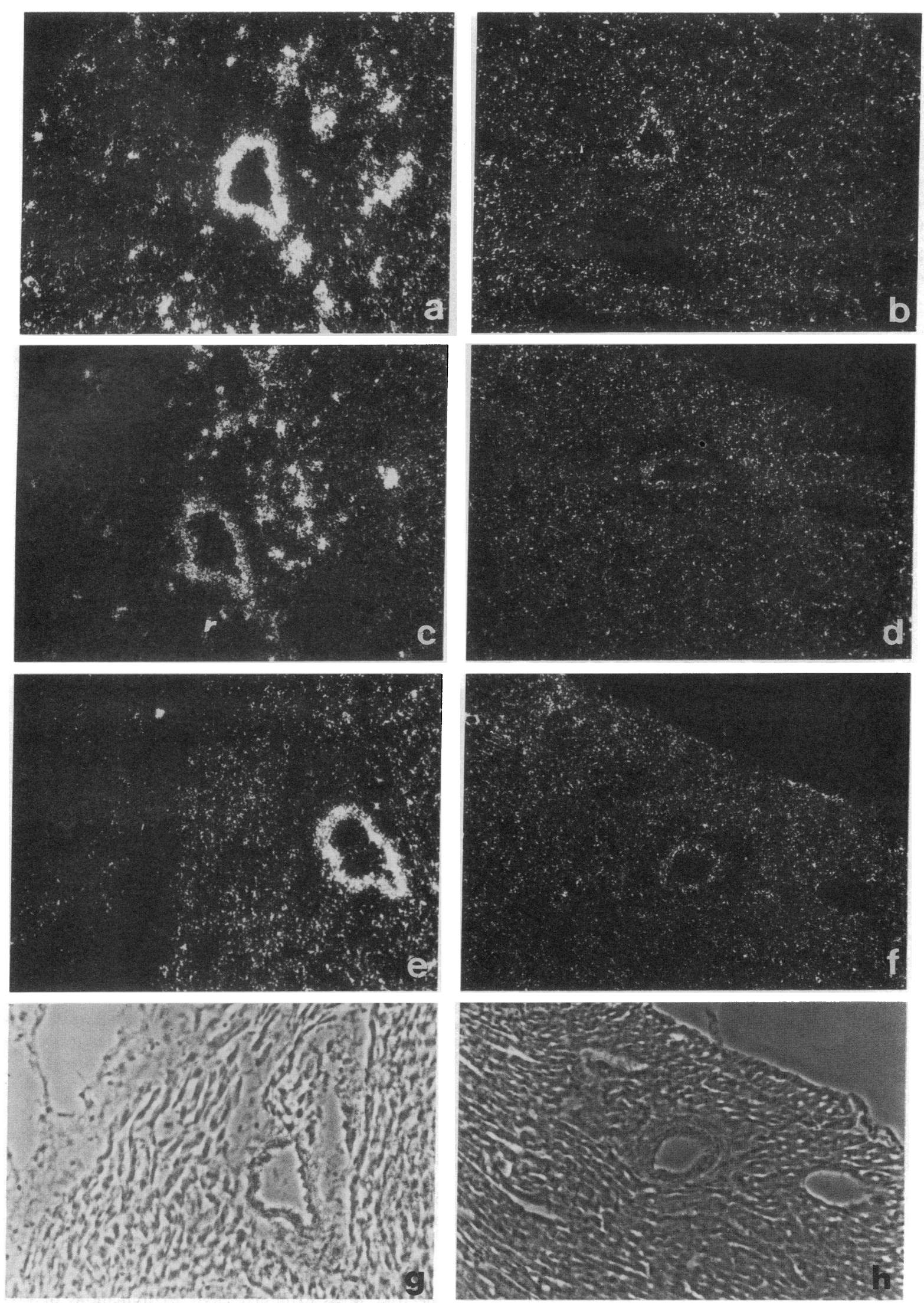

Figure 4. FN mRNAs accumulate within rat myocardium as early as $1 \mathrm{~d}$ after thoracic aortic stenosis. Serial sections of ventricle from operated $(a, c, e, g)$ and normal animals $(b, d, f, h)$ were hybridized with FN-I $(a, b)$, -EIIIA $(c, d)$, -EIIIB probes $(e, f)$. A strong signal is obtained with all the probes in the coronary wall of operated animal while only a weak signal is detected with FN-I probes within the coronary arteries of normal heart. Note the absence of FN-EIIIB mRNAs and the presence of FN-EIIIA and -I mRNAs within the myocardium $1 \mathrm{~d}$ after aortic stenosis. 

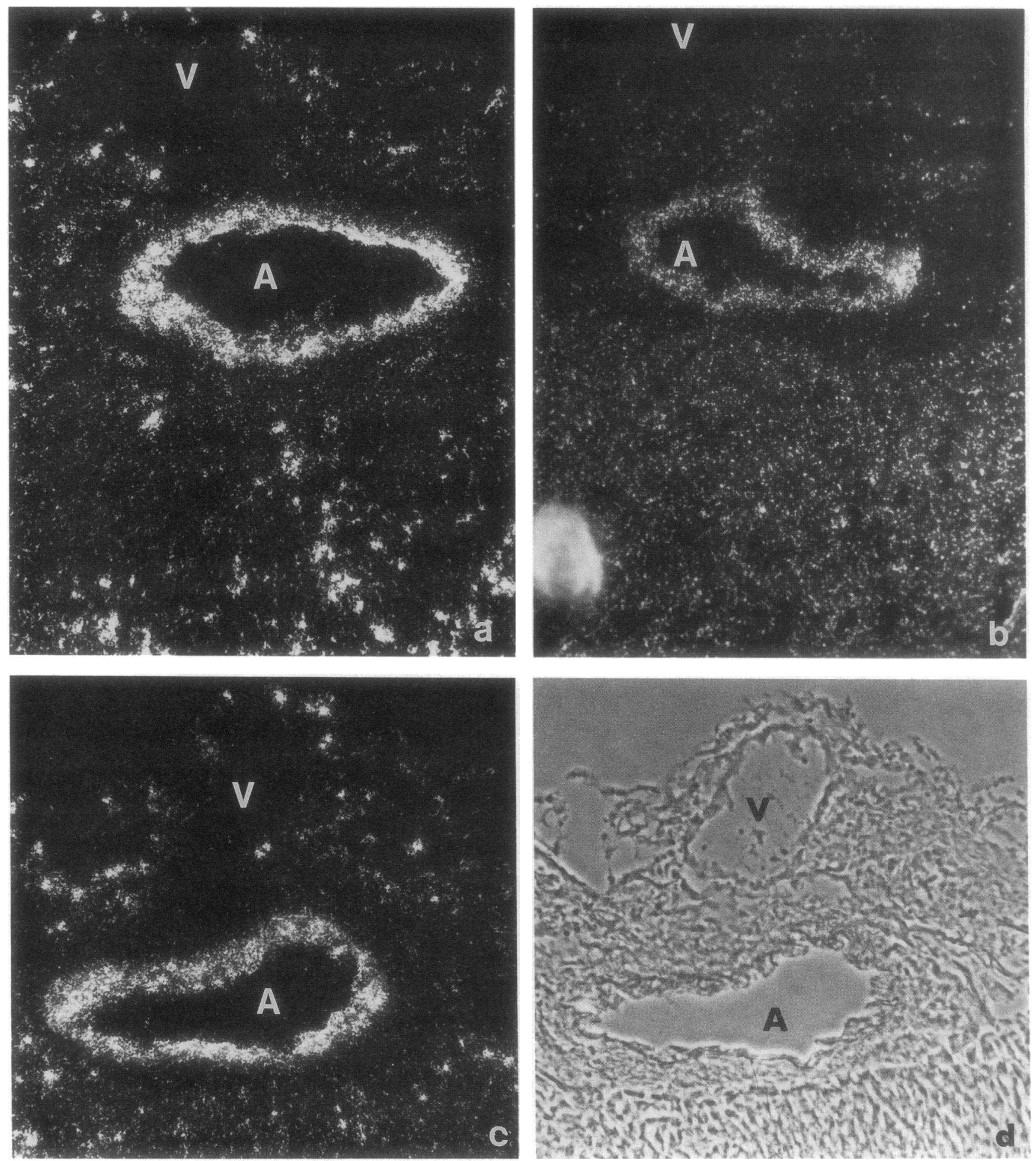

Figure 5. Accumulation of FN mRNAs within the media of large arteries. Serial nonconsecutive sections of the left ventricle at day 1 after aortic stenosis hybridized with FN-I $(a)$, EIIIB $(b)$, EIIIA $(c)$. Note the FN mRNAs accumulation within arterial wall $(A)$ while they are absent from the vein $(V)$.

FN probes and were identical to normal unoperated 25-d-old controls (Fig. 4).

By day 3, the degree of hypertrophy was $20 \%$ and some focal areas of necrosis could be found in the left ventricle by phase contrast analysis (Fig. 6) as previously described $(14,24)$.
These necrotic areas exhibited large accumulations of FNEIIIB and -EIIIA mRNAs, whereas the normal myocardium was completely unlabeled. The accumulation of both mRNAs in the damaged myocardium was spatially coordinated. At this time, the media of some arteries was reactive to FN-EIIIA and 

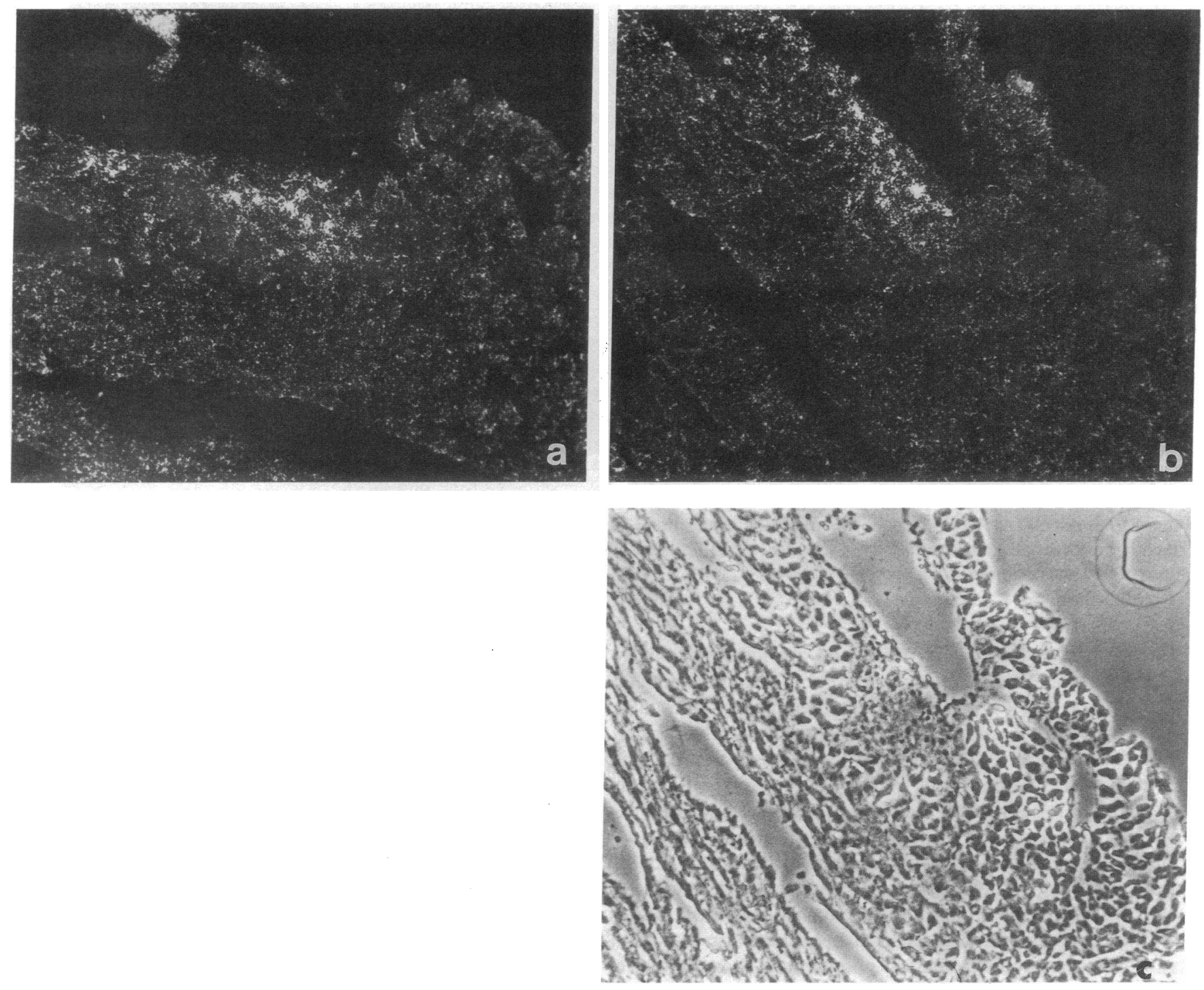

Figure 6. Expression of FN-EIIIA and -EIIIB mRNA within fibrotic areas of the myocardium, $3 \mathrm{~d}$ after aortic stenosis. Serial sections of the left ventricle were hybridized with EIIIA $(a)$ and EIIIB $(b)$ probes, in $(c)$ phase contrast image.

-EIIIB probes. However, no correlation between the vascular and the myocardial reactivity was observed. By day 4 , when hypertrophy reached 35\% FN mRNA distribution was similar to that observed at day 3. Coronary arteries located in the right ventricle appear to strongly react with the FN-I probe as exemplified in Fig. 7.

The specificity of each probe was confirmed by the absence of labeling when sense probes synthesized from the same construct were used for hybridization. One example is illustrated in Fig. $7 b$.

\section{Discussion}

The results of the present study demonstrate that $(a)$ the cardiac expression of FN-EIIIA ${ }^{+}$and -EIIIB ${ }^{+}$mRNA is developmentally regulated being high in fetus and undetectable in adult; (b) FN-EIIIA ${ }^{+}$and -EIIIB ${ }^{+}$mRNAs are reexpressed in defined areas of the rat heart and in the coronary arteries very early after aortic stenosis. Therefore, the accumulation of FN-
EIIIA $^{+}$and EIIIB ${ }^{+}$mRNAs soon after the imposition of pressure overload must reflect the induction of the genomic program of nonmuscle cells and smooth muscle cells towards a fetal profile. It is the first demonstration of an accumulation of FN mRNAs in the coronary arteries as early as $1 \mathrm{~d}$ after imposition of increased perfusion pressure, suggesting that mechanical factors play a major role in the induction of FN gene as a result of mechanical overload.

FN mRNA splicing is developmentally regulated (11) and tissue-specific alternative splicing of mRNAs may constitute an important mechanism of genetic regulation during development, as it has been proposed for other genes $(28,29)$. Indeed both EIIIA and EIIIB domains are coexpressed in the early embryo and are spliced out in a tissue-specific manner after the completion of embryogenesis and organogenesis $(10,11)$. For example, the pattern of FN mRNA splicing was recently shown to exhibit an organ-specific pattern during fetal and postnatal development of human smooth muscle $(12,30)$. In this paper we demonstrate that at the latest stage of fetal development, the two embryonic variants of FN mRNAs $\left(\right.$ EIIIA $^{+}$and EIIIB $^{+}$) are 

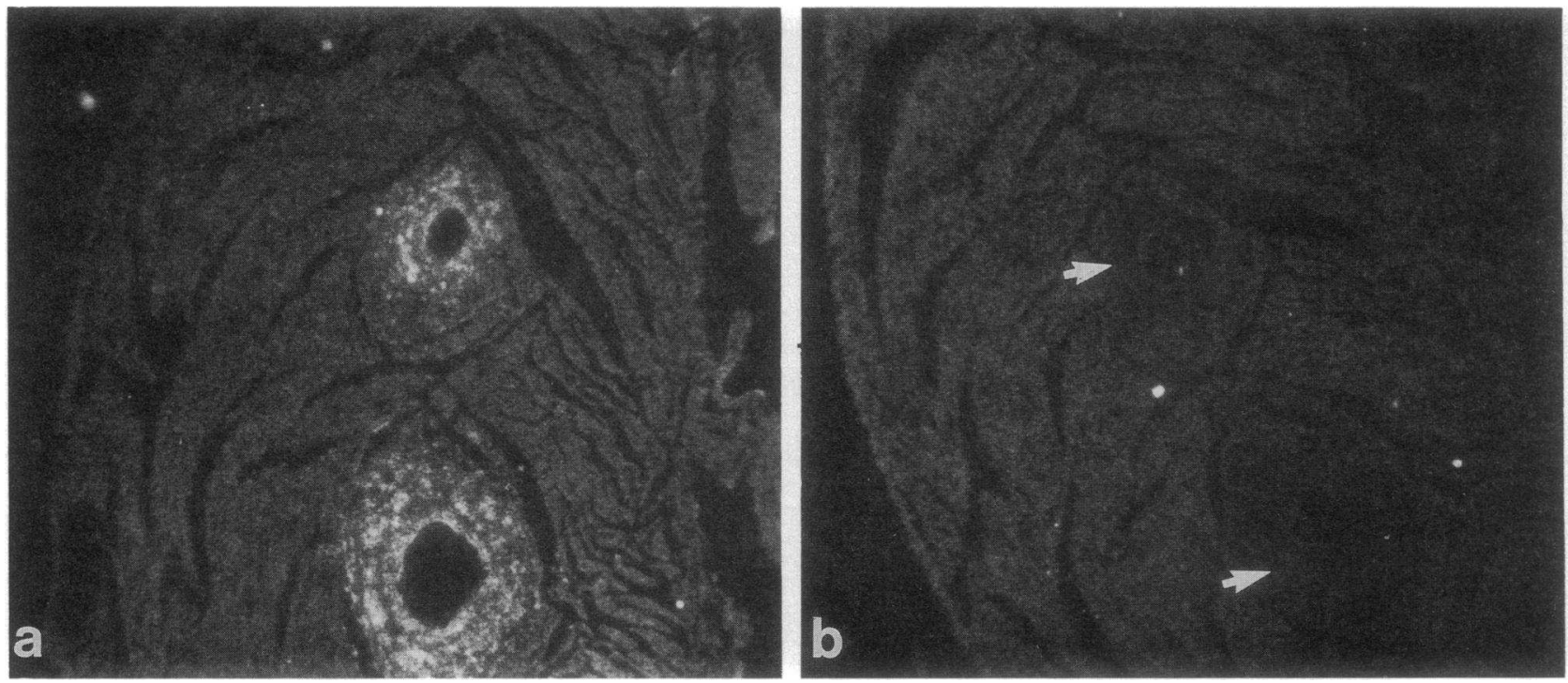

Figure 7. Specificity of FN-I probes in hypertrophied myocardium. Serial sections of the right ventricle of a heart submitted for $4 \mathrm{~d}$ to aortic stenosis were hybridized with antisense $(a)$ or sense $(b)$ FN-I probes. Note the very low background obtained with sense probes (arrows) in the artery wall when compared to the positive signal $(a)$.

still accumulated in the rat heart and are localized mainly within the nonmuscle cells such as fibroblasts and endothelial cells. These mRNAs are most abundant within trabeculae where myocardial tissue is still growing (31), but they are almost undetectable in the midwall where the tissue has become well organized. Furthermore, in the 3-wk-old rats in which cardiac growth is completed, the FN gene is either no longer expressed, or is expressed at a very low level. Thus the developmental program of differential genomic expression is not restricted to some proteins expressed by myocytes (review in 15) but also involves a protein of the extracellular matrix that is synthesized by nonmuscle cells. Our results are also consistent with the immunohistochemical observations showing that cFN and particularly FN-EIIIA ${ }^{+}$is barely detectable within both cardiac muscle and large vessels of the adult, while p-FN is abundant $(12,13,30)$, suggesting that part of the fibronectin present in adult cardiac extracellular matrix may be provided by the circulating $\mathrm{p}-\mathrm{FN}$. The fact that in cardiac tissue $(a)$ the expression of the fibronectin gene is developmentally regulated, and $(b)$ the time course of accumulation of the FNEIIIA $^{+}$and -EIIIB ${ }^{+}$variants differs from that of other muscles $(12,30)$ suggests a role for these variants in the active proliferation and migration of cells during cardiac development as has been proposed in other tissues at earlier stages of ontogenesis (32). This developmental regulation of FN splicing in the heart must also be related to changes in the adhesive properties of the striated myocytes to ECM proteins. Indeed myocytes from neonatal hearts adhere to FN substrate while adult cells do not (33). Further studies will determine whether changes in the FN phenotype are related to specific changes in the types of FN receptors appearing during the ontogenic development of the heart.

An increase in extracellular matrix proteins, such as FN and collagen, and of their corresponding mRNAs, has been associated with the development of cardiac hypertrophy (14, $17,34,35)$. In a previous report, we described an accumulation of immunoreactive $\mathrm{FN}$ in necrotic areas of the left ventricular myocardium, as early as $2 \mathrm{~d}$ after aortic stenosis (14). Using this immunological approach, it was not possible to distinguish between an uptake of p-FN from the blood or increased synthesis and secretion of FN by cardiac cells. In this study we demonstrate that during the same period there is an accumulation of FN mRNAs, implying that FN is synthesized and secreted locally soon after cardiac overload. Our results do not exclude the possibility that an exsudate of p-FN also accumulates in the extracellular matrix during the fibrotic process (36).

The response of the heart to pressure overload is characterized by the reexpression of the fetal genetic program for several proteins of the myocyte $(15,16,37)$. Here we show a reexpression of the fetal forms of FN mRNAs by nonmuscle cells. The expression of the two fetal mRNAs is not temporally coordinated; indeed only the EIIIA ${ }^{+}$mRNA variant is observed in the myocardium within $24 \mathrm{~h}$ after pressure overload. At later times ( $3 \mathrm{~d}$ after aortic stenosis) when significant hypertrophy has occurred, both EIIIA ${ }^{+}$and EIIIB ${ }^{+}$mRNAs accumulate, primarily within areas of myocardial damage where reparative fibrosis occurs $(14,34)$. These results suggest that the expression of these two variants may depend upon the severity of the lesion. Thus the heart responds to injury by altering the splicing of FN mRNA to give rise to the variant normally expressed in fetal tissue. The localization of embryonic mRNA variants in the damaged myocardium is consistent with a role played by these variants in cell migration and proliferation or chemotaxis in wound healing (38) and may be involved in myocardium repair. As suggested (38), FN splicing may be used in cardiac muscle as a mechanism to create functionally appropriate forms of FN that facilitate the process of fibrosis.

The increased expression of the FN gene in suffering myocardium might be secondary to an increased production of TGF $\beta$ mRNA resulting in TGF $\beta$ accumulation within areas of ischemia (39). TGF $\beta$ has been shown to favor the formation of EIIIA $^{+}$FN (40). It seems likely that this and other growth factors are involved in the localized upregulation of the FN gene in the hypertrophying heart. 
The demonstration that FN mRNAs accumulate within the inner part of the coronary arteries as early as 1 day after aortic stenosis is a major finding in this study. The upregulation of the fibronectin gene with the reexpression of the embryonic mRNA profile constitutes one of the earliest responses of the arterial cells to a sudden increase in arterial pressure. Thickening of the arterial wall is a well-known feature of hypertensive diseases and the thickness of the coronary wall is a criterium of cardiac hypertrophy (34). Increase in the expression of the FNEIIIA $^{+}$variant has recently been shown to characterize arterial lesions such as thickening of the intima and the atherosclerotic plaque (30). Because no intimal thickening or atherosclerotic lesions have been described in rat cardiac vessels after aortic stenosis, we postulate that the accumulation of both EIIIA ${ }^{+}$ and EIIIB $^{+}$mRNAs in arteries is not related to the inflammatory lesions associated with damaged intima; they must be the result of the hypertrophic and hyperplasic processes which develop in the arterial media in response to the imposed increased pressure. Because FN mRNAs accumulate only in arteries and not in veins even when the two vessels are closely located (Fig. 5), we assume that only local and cell-specific factors secondary to perfusion pressure trigger the upregulation of the FN gene. Interestingly, the accumulation of the fetal form of the MHC mRNAs $(\beta-\mathrm{MHC})$, one of the major changes in the genomic expression of myocytes, also occurs first in the vicinity of large arteries (24) with a time course of appearance similar to that observed here for the FN mRNAs. Both mechanical and humoral factors may be implicated in the induction of the $\beta$ MHC gene (41). Indeed, passive stretch has been proposed as a major mechanical factor implicated in the regulation of the $\beta$-MHC gene in vivo (24) and both TGF $\beta$ and basic FGF can induce $\beta$-MHC gene expression in vitro (42). The trigger for the upregulation of the FN gene in arteries may be vascular stretch, alone or combined with growth factors such as TGF $\beta$ or other hormonal peptides (34). Indeed an initial trigger such as endothelial stretch induced by increase coronary perfusion pressure may induce a cascade of events such as the synthesis of endothelin and/or EDRF which would activate the secretion of $\operatorname{TGF} \beta(34,43,44)$. Therefore, it can be hypothesized that the same initial events are involved in the upregulation of multigene families such as MHC genes or activation of single genes giving rise to multiple mature RNAs by alternative splicing.

In the rat heart submitted to a sudden pressure overload, the FN gene is upregulated at the early stage of the development of cardiac hypertrophy and exhibits a unique pattern of expression since mRNAs containing the exons specific to the embryonic variants are coexpressed within both smooth and striated muscle tissue. FN-EIIIA ${ }^{+}$and -EIIIB ${ }^{+}$mRNAs accumulate in areas of the heart where an important tissular remodeling is occurring. The expression of embryonic mRNAs is associated with different processes such as growth, hypertrophy, and repair of the myocardium. It remains to assess if the regulation of RNA splicing depends more on a cellular stretch or on a hormonal regulation that is associated with cardiac growth.

In conclusion it appears that expression of the fetal mRNA profile in response to increased pressure in the rat heart is a feature common to muscle and nonmuscle cells.

\section{Acknowledgments}

The authors wish to thank B. Swynghedauw, J. J. Mercadier, and G. Buttler Brown for helpful and stimulating discussion.
This work was supported by INSERM and CNRS. A. Barrieux was a recipient of a grant from Foundation de la Recherche Medicale. $V$. Koteliansky was a recipient from the Ministère de la Recherche et de la Technologie.

\section{References}

1. Ahumada, G. G., and J. E. Saffitz. 1984. Fibronectin in rat heart. A link between cardiac myocytes and collagen. J. Histochem. Cytochem. 32:383-388.

2. Oh, E., M. Pierschbacher, and E. Ruoslati. 1981. Deposition of plasma fibronectin in tissues. Proc. Natl. Acad. Sci. USA. 78:3218-3221.

3. Tamkun, J. W., and R. O. Hynes. 1983. Plasma fibronectin is synthesized and secreted by hepatocytes. J. Biol. Chem. 258:4641-4646.

4. Borsi, L., B. Carnemolla, P. Castellani, C. Rosellini, D. Vecchio, G. Allemanni, S. E. Chang, J. Taylor-Papadimitriou, H. Pande, and L. Zardi. 1987 Monoclonal antibodies in the analysis of fibronectin isoforms generated by alternative splicing of mRNA precursors in normal and transformed cells. J. Cell Biol. 104:595-600.

5. Vartio, T., L. Laitinen, O. Narvanen, M. Cutolo, L. E. Thornell, L. Zardi, and I. Virtanen. 1987. Differential expression of the ED sequence-containing form of cellular fibronectin in embryonic and adult human tissues. J. Cell Sci. 88:419-430.

6. Hynes, R. O. 1985. Molecular biology of fibronectin. Annu. Rev. Cell. Biol. 1:67-90.

7. Petersen, T. E., K. Skorstengaard, and K. Vibe-Petersen. 1989. Primary structure of fibronectin. In Fibronectins D. F. Mosher, editor. Academic Press, Inc., New York. 1-24.

8. Gutman, A., and A. R. Kornblitt. 1987. Identification of a third region of cell-specific alternative splicing in human fibronectin mRNA. Proc. Natl. Acad. Sci. USA. 84:7179-7182.

9. Schwarzbauer, J. E., R. S. Patel, D. Fonda, and R. Hynes. 1987. Multiple sites of alternative splicing of the rat fibronectin gene transcript. EMBO (Eur. Mol. Biol. Organ.) J. 6:2573-2580.

10. ffrench-Constant, C., and R. O. Hynes. 1988. Pattern of fibronectin gene expression and splicing during cell migration in chicken embryos. Development. 104:369-382.

11. ffrench-Constant, C., and R. O. Hynes. 1989. Alternative splicing of fibronectin is temporally and spatially regulated in chicken embryos. Development (Comb.). 106:375-388.

12. Glukhova, M. A., M. G. Frid, B. V. Shekhonin, Y. V. Balabanov, and V. E. Koteliansky. 1990. Expression of fibronectin variants in vascular and visceral smooth muscle cell in development. Dev. Biol. 140:193-202.

13. Shekhonin, B. V., S. B. Guriev, S. B. Irgashev, and V. E. Koteliansky. 1990. Immunofluorescent identification of fibronectin and fibrinogen/fibrin in experimental myocardial infarction. J. Mol. Cell. Cardiol. 22:533-541.

14. Contard, F., V. Koteliansky, F. Marotte, I. Dubus, L. Rappaport, and J. L. Samuel. 1991. Specific alterations in the distribution of extracellular matrix components within rat myocardium during development of pressure overload. $L a b$. Invest. 64:65-75.

15. Lompré, A. M., J. J. Mercadier, and K. Schwartz. 1990. Changes in gene expression during cardiac growth. Int. Cell Rev. 124:137-186.

16. Schwartz, K., J. J. Mercadier, B. Swynghedauw, and A. M. Lompré. 1988. Modifications of gene expression in cardiac hypertrophy. Heart Failure. 4:154163.

17. Chapman, D., K. T. Weber, and M. Eghbali. 1990. Regulation of fibrillar collagen types I and III and basement membrane type IV collagen gene expression in pressure overloaded rat myocardium. Circ. Res. 67:787-794.

18. Eghbali, M., M. Czaja, F. R. Zeydel, M. A. Weiner, S. Zern, S. Seifter, and O. O. Blumenfeld. 1988. Collagen chain mRNAs in isolated heart cells from young and adult rats. J. Mol. Cell. Cardiol. 20:267-276.

19. Terracio, L., and T. K. Borg. 1988. Factors affecting cardiac cell shape. Heart Failure. 4:114-124.

20. Eghbali, M., O. O. Blumenfeld, S. Seifter, P. M. Buttrick, L. A. Leinwand, T. F. Robinson, M. A. Zern, and M. A. Giambrone. 1989. Localization of types I, III and IV collagen mRNAs in rat heart cells by in situ hybridization. J. Mol. Cell. Cardiol. 21:103-113.

21. Bugaisky, L. B., E. Siegel, and R. J. Whalen. 1983. Myosin isozyme changes in the heart following constriction of the ascending aorta of a 25 day old rat. FEBS (Fed. Eur. Biochem. Soc.) Lett. 161:230-234.

22. Kornblitt, A. R., K. Vibe Pedersen, and F. Baralle. 1984. Human fibronectin: molecular cloning evidence for two mRNA species differing by an internal segment coding for a structural domain. EMBO (Eur. Mol. Biol. Organ.) J. 3:221-226.

23. Dufour, S., A. Gutman, F. Bois, N. Lamb, J. P. Thiery, and A. R. Kornblitt. 1991. Generation of full-length cDNA recombinant vectors for the transient expression of human fibronectin in mammalian cell lines. Exp. Cell. Res. 193:331-338

24. Schiaffino, S., J. L. Samuel, D. Sassoon, A. M. Lompré, I. Garner, F. 
Marotte, M. Buckingham, L. Rappaport, and K. Schwartz. 1989. Nonsynchronous accumulation of $\alpha$-skeletal actin and $\beta$-myosin heavy chains during the early stages of pressure-overload-induced cardiac hypertrophy demonstrated by in situ hybridization. Circ. Res. 64:937-948.

25. Wilkinson, D. G., J. A. Bailes, and A. P. McMahon. 1987. Expression of the proto-oncogene int-1 is restricted to specific neural cells in the developing rat embryo. Cell. 50:79-88.

26. Norton, P. A., and R. O. Hynes. 1987. Alternative splicing of chicken fibronectin in embryos and in normal and transformed cells. Mol. Cell. Biol. 7:4297-4307.

27. Delcayre, C., J. L. Samuel, F. Marotte, M. Best-Belpomme, J. J. Mercadier, and L. Rappaport. 1988. Synthesis of stress proteins in rat cardiac myocytes 2-4 days after imposition of hemodynamic overload. J. Clin. Invest. 82:460-468.

28. Andreadis, A., M. E. Gallego, and B. Nadal-Ginard. 1987. Generation of protein isoform diversity by alternative splicing: mechanism and biological implications. Annu. Rev. Cell Biol. 3:204-242.

29. Breitbart, R. E., and B. Nadal-Ginard. 1987. Developmentally induced muscle-specific trans factors control the differential splicing of alternative and constitutive troponin T exons. Cell. 49:793-803.

30. Glukhova, M. A., M. G. Frid, B. V. Shekhonin, T. D. Vasilevskaya, J. Grunwald, M. Saginati, and V. E. Koteliansky. 1989. Expression of extra domain A fibronectin sequence in vascular smooth muscle type is phenotype dependent. J. Cell Biol. 109:357-363.

31. Van Mierop, L. H. S. 1979. Morphological development of the heart. In Handbook of Physiology. Section 2. The Cardiovascular System. R. M. Berne, editor. American Physiology Society, Bethesda, MD. 29-42.

32. Dufour, S., J. L. Duband, A. R. Kornblitt, and J. P. Thiéry. 1988. The role of fibronectin in embryonic cell migration. Trends Genet. 4:198-203.

33. Borg, T. K., R. Kristofer, E. Lundgren, K. Borg, and B. Obrink. 1984 Recognition of extracellular matrix components by neonatal and adult cardiac myocytes. Dev. Biol. 104:86-94.
34. Brilla, C. G., R. Pick, L. B. Tan, J. S. Janicki, and K. T. Weber. 1990. Remodelling of the rat right and left ventricles in experimental hypertension. Circ. Res. 67:1355-1364.

35. Villareal, F. J., J. W. Cowell, and W. H. Dillmann. 1990. Changes in gene expression of extra cellular matrix proteins with myocardial hypertrophy. J. Cell Biol. 111:147a. (Abstr.)

36. Kuhn III, C., J. Bolt, T. E. King, E. Crouch, T. Vartio, and J. A. McDonald. 1989. An immunohistochemical study of architectural remodeling and connective tissue synthesis in pulmonary synthesis. Am. Rev. Respir. Dis. 140:16931703.

37. Nadal-Ginard, B., and V. Mahdavi. 1989. Molecular basis of cardiac performance. J. Clin. Invest. 84:1693-1700.

38. ffrench-Constant, C., L. Van De Water, H. F. Dvorak, and R. O. Hynes. 1989. Reappearance of an embryonic pattern of fibronectin splicing during wound healing in the adult rat. J. Cell Biol. 109:903-914.

39. Thompson, N. L., F. Bazoberry, E. H. Speir, W. Casscells, V. J. Ferrans, K. C. Flanders, P. Kondaiah, A. G. Geiser, and M. B. Sporn. 1988. Transforming growth factor beta-1 in acute myocardial infarction in rats. Growth Factors. 1:9199.

40. Balza, E., L. Borsi, G. Allemani, and L. Zardi. 1988. Transforming growth factor $\beta$ regulates the levels of different fibronectin isoforms in normal human cultured fibroblasts. FEBS (Fed. Eur. Biochem. Soc.) Lett. 228:42-44.

41. Samuel, J. L. I. Dubus, F. Contard, K. Schwartz, and L. Rappaport. 1990. Biological signals of cardiac hypertrophy. Eur. Heart J. 11(Suppl. G):1-7.

42. Parker, T. G., S. E. Parker, and M. D. Schneider. 1990. Peptide growth factors provoke "fetal" contractile protein gene expression in rat cardiac myocytes. J. Clin. Invest. 85:507-514.

43. Vane, J. 1990. Endothelins come home to roost. Nature (Lond.). 348:673. 44. Lefer, A. M., P. Tsao, N. Aoki, and M. A. Palladino. 1990. Mediation of cardioprotection by transforming growth factor- $\beta$. Science (Wash. DC). 249:6163. 